\title{
The Galactic Disk-Halo Transition - Evidence from Stellar Abundances $\dagger$
}

\author{
Poul Erik Nissen ${ }^{1}$ and William J. Schuster ${ }^{2}$ \\ ${ }^{1}$ Department of Physics and Astronomy, University of Aarhus, DK-8000 Aarhus C, Denmark \\ email: pen@phys.au.dk \\ ${ }^{2}$ Observatorio Astronómico Nacional, Universidad Nacional Autónoma de México, Apartado \\ Postal 877, Ensenada, BC, 22800 México \\ email: schuster@astrosen.unam.mx
}

\begin{abstract}
New information on the relations between the Galactic disks, the halo, and satellite galaxies is being obtained from elemental abundances of stars having metallicities in the range $-1.5<[\mathrm{Fe} / \mathrm{H}]<-0.5$. The first results for a sample of 26 halo stars and 13 thick-disk stars observed with the ESO VLT/UVES spectrograph are presented. The halo stars fall in two distinct groups: one group (9 stars) has $[\alpha / \mathrm{Fe}]=0.30 \pm 0.03$ like the thick-disk stars. The other group (17 stars) shows a clearly deviating trend ranging from $[\alpha / \mathrm{Fe}]=0.20$ at $[\mathrm{Fe} / \mathrm{H}]=-1.3$ to $[\alpha / \mathrm{Fe}]=0.08$ at $[\mathrm{Fe} / \mathrm{H}]=-0.8$. The kinematics of the stars are discussed and the abundance ratios $\mathrm{Na} / \mathrm{Fe}, \mathrm{Ni} / \mathrm{Fe}, \mathrm{Cu} / \mathrm{Fe}$ and $\mathrm{Ba} / \mathrm{Y}$ are applied to see if the "low-alpha" stars are connected to the thin disk or to Milky Way satellite galaxies. Furthermore, we compare our data with simulations of chemical abundance distributions in hierarchically formed stellar halos in a $\Lambda$ CDM Universe.
\end{abstract}

Keywords. stars: abundances, Galaxy: disk, Galaxy: halo, Galaxy: evolution

\section{Introduction}

As discussed by Venn et al. (2004), the chemical signatures of stars in Milky Way dwarf spheroidal (dSph) galaxies are different from the majority of Galactic thin-disk, thick-disk and halo stars. The dSph stars have lower values of $\alpha / \mathrm{Fe}$, where $\alpha$ refers to the abundance of typical alpha-capture elements like $\mathrm{Mg}, \mathrm{Si}, \mathrm{Ca}$ and $\mathrm{Ti}$, than the Galactic stars. Furthermore, the Ba/Y ratio is significantly higher in the dSph stars. Thus, Venn et al. conclude that the main components of our Galaxy have not been formed through the merging of dwarf galaxies similar to present-day dSphs. This could be seen as an argument against hierarchical structure formation as predicted in Cold Dark Matter cosmologies. Font et al. (2006) have, however, made $\Lambda$ CDM simulations of the chemical abundance distributions in the the Galactic halo, which show that early accreted dwarf galaxies have chemical properties different from those of present-day satellite galaxies. For the metal-poor part of the Galactic halo, they predict enhanced values of $[\alpha / \mathrm{Fe}]$ corresponding to the ratio calculated for Type II supernovae, but for the metal-rich end of the halo a decline of $[\alpha / \mathrm{Fe}]$ is predicted due to an increasing contribution of iron from Type Ia SNe.

In order to test these recent simulations, we have started a survey of abundance ratios of about 100 halo stars having $-1.5<[\mathrm{Fe} / \mathrm{H}]<-0.5$. This metallicity range also contains thin-disk and thick-disk stars, and hence a comparison of abundances may give new information on the relations between the various Galactic populations.

$\dagger$ Based on observations made with the ESO Telescopes at the Paranal Observatory under programs 65.L-0507, 67.D-0439, 68.D-0094, 68.B-0475, 69.D-0679, 70.D-0474, and 76.B-0133. 
The survey is a continuation of a more limited study by Nissen \& Schuster (1997), who determined abundances of 13 halo stars and 16 thick disk stars in the metallicity range $-1.3<[\mathrm{Fe} / \mathrm{H}]<-0.4$. Interestingly, 8 of the halo stars turned out to have significantly lower values of $[\alpha / \mathrm{Fe}] \dagger$ than the other halo stars. Considering the small number of halo stars studied it is, however, unclear from the work of Nissen \& Schuster if the distribution of $[\alpha / \mathrm{Fe}]$ in the halo is continuous or bimodal. One of the aims of the present study is to answer this question by observing a larger sample of stars.

\section{Selection of stars and observations}

The stars have been selected from Schuster et al. (2006), which contains uvby- $\beta$ photometry and complete kinematic data for 1533 high-velocity and metal-poor stars. In order to ensure that a selected star belongs to the halo population, we required that its total space velocity with respect to the Local Standard of Rest (LSR), $V_{\text {total }}$, should be larger than $180 \mathrm{~km} \mathrm{~s}^{-1}$. Furthermore, we used the Strömgren indices, $b-y, m_{1}$ and $c_{1}$, to select dwarfs and subgiants with temperatures $5100<T_{\text {eff }}<6200 \mathrm{~K}$ and metallicities $-1.5<[\mathrm{Fe} / \mathrm{H}]<-0.5$. When selecting the stars from the bright end of the Schuster et al. catalogue, these criteria result in a limiting magnitude of $V=11.1$ for stars that could be reached with the Nordic Optical Telescope (NOT) on La Palma.

The first observations with NOT and its FIbre fed Echelle Spectrograph (FIES) were carried out in May 2008 and resulted in high resolution $(R \sim 45000)$ spectra with $S / N \sim 150$ for 30 halo stars. The abundance analysis of these data is, however, not yet finished. Instead, we are presenting here results for 26 halo stars that fulfil our selection criteria, and for which spectra obtained with the VLT/UVES spectrograph are available in the ESO science archive. These spectra have $R \sim 60000$ and very high signal-to-noise ratios, $S / N>300$, in the 4800-6500 $\AA$ region that we are using. In addition, 13 thick-disk stars with UVES spectra are included. Their atmospheric parameters fall in the same ranges as the halo stars, but their total space velocities relative to the LSR are typical for thick-disk stars $\left(50<V_{\text {total }}<150 \mathrm{~km} \mathrm{~s}^{-1}\right)$.

\section{Abundance analysis}

Abundances of the elements $\mathrm{Mg}$, Si, Na, Ca, Ti, Cr, Ni, Cu, Y and $\mathrm{Ba}$ are derived from equivalent widths of weak to medium-strong lines $(5<E W<80 \mathrm{~m} \AA)$. A grid of 1D MARCS model-atmospheres is used to derive the abundances, and the effect on the electron pressure from variations of $[\alpha / \mathrm{Fe}]$ is taken into account. Like Nissen \& Schuster (1997), the analysis is performed in a differential way with respect to two bright thickdisk stars, HD 22879 and HD 76932, that are known to have $[\alpha / \mathrm{Fe}]$ close to +0.3 dex according to several studies (e.g. Reddy et al. 2006). For nearby stars that appear to be unreddened, as judged from the absence of interstellar NaD lines, $T_{\text {eff }}$ is derived from the $b-y$ and $V-K$ color indices, and the gravity parameter, $\log g$, is estimated via the Hipparcos parallax. For the more distant and reddened stars, $T_{\text {eff }}$ is determined from the excitation balance of Fe I lines, and $\log g$ is derived from the requirement that the difference in Fe abundances derived from Fe II and Fe I lines should be the same as in HD 22879 and HD 76932. The adopted metallicity, [Fe/H], is that derived from Fe II lines.

In order to minimize the dependence of abundance ratios on possible errors in the atmospheric parameters, the abundance ratio of two elements is derived from lines belonging to the same ionization stage, e.g. $[\mathrm{Mg} / \mathrm{Fe}]$ from $\mathrm{Mg}$ I and $\mathrm{Fe}$ I lines or $[\mathrm{Ba} / \mathrm{Y}]$ from

$\dagger$ Defined as $[\alpha / \mathrm{Fe}]=\frac{1}{4}([\mathrm{Mg} / \mathrm{Fe}]+[\mathrm{Si} / \mathrm{Fe}]+[\mathrm{Ca} / \mathrm{Fe}]+[\mathrm{Ti} / \mathrm{Fe}])$ 

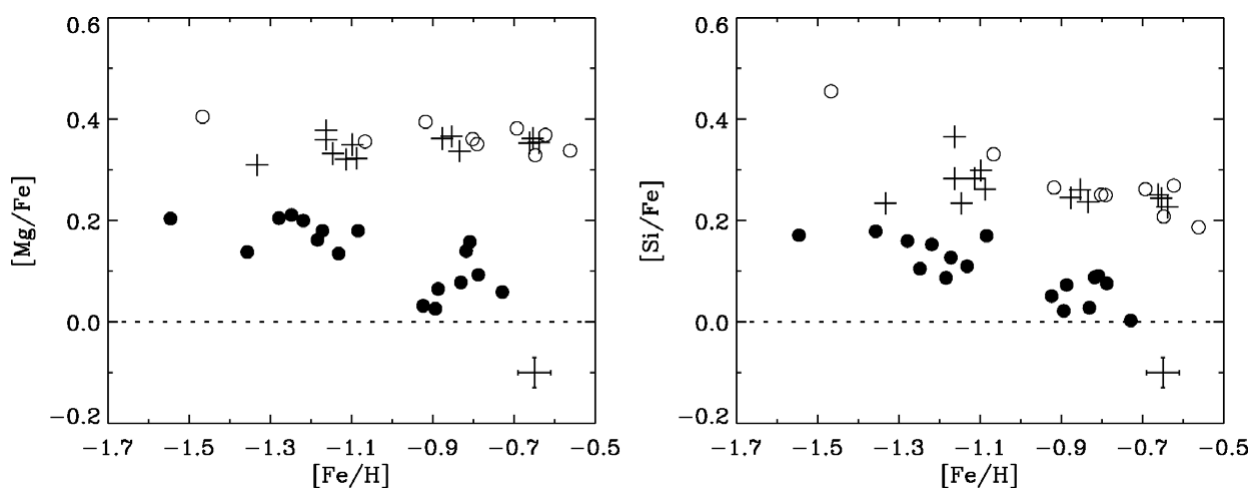

Figure 1. $[\mathrm{Mg} / \mathrm{Fe}]$ and $[\mathrm{Si} / \mathrm{Fe}]$ vs. $[\mathrm{Fe} / \mathrm{H}]$ for the sample of stars with VLT/UVES spectra. Crosses: Thick-disk stars; Open circles: "High-alpha" halo stars; Filled circles: "Low-alpha" halo stars. Typical error bars for the data are shown in the lower right corners of the figures.
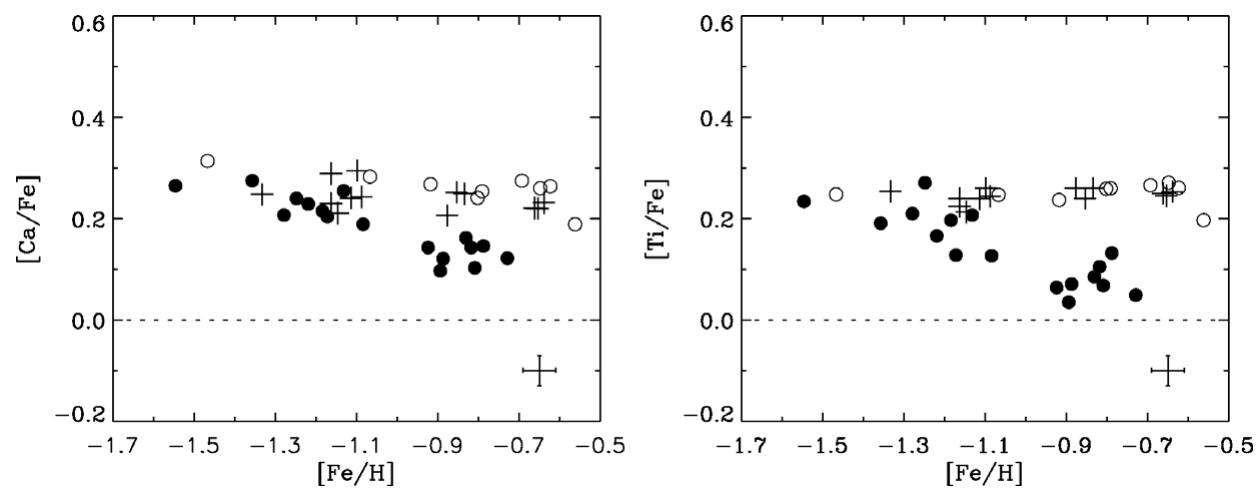

Figure 2. $[\mathrm{Ca} / \mathrm{Fe}]$ and $[\mathrm{Ti} / \mathrm{Fe}]$ vs. $[\mathrm{Fe} / \mathrm{H}]$. Same symbols as in Fig. 1.

Ba II and Y II lines. Local thermodynamic equilibrium (LTE) is assumed, but due to the limited range of the parameters of our stars, non-LTE effects on the derived differential abundances are expected to be small.

\section{Results and discussion}

Figures 1 and 2 show the derived abundances of the four alpha-capture elements, $\mathrm{Mg}$, $\mathrm{Si}, \mathrm{Ca}$ and $\mathrm{Ti}$, relative to $\mathrm{Fe}$. As seen, the halo stars fall in two distinct groups: $i$ ) the "high-alpha" stars that have very near the same $[\alpha / \mathrm{Fe}]$ as the thick-disk stars and a remarkably small scatter, $\pm 0.03 \mathrm{dex}$, around the "plateau" value of $[\alpha / \mathrm{Fe}]$ and $i i)$ the "low-alpha" stars that show a decreasing trend of $[\alpha / \mathrm{Fe}]$ as a function of increasing $[\mathrm{Fe} / \mathrm{H}]$. In the cases of $\mathrm{Mg}$ and $\mathrm{Si}$ the separation of the two groups of halo stars is seen for the whole range $-1.5<[\mathrm{Fe} / \mathrm{H}]<-0.7$ with a maximum separation of $\sim 0.25 \mathrm{dex}$ in $[\mathrm{Mg} / \mathrm{Fe}]$ and $\sim 0.20$ dex in $[\mathrm{Si} / \mathrm{Fe}]$. For $\mathrm{Ca}$ and Ti the two groups tend to merge at $[\mathrm{Fe} / \mathrm{H}]$ $\simeq-1.2$, and the maximum separation is only about 0.12 dex for $\mathrm{Ca}$ and about $0.17 \mathrm{dex}$ for Ti.

A possible explanation of these trends is that the "high-alpha" and the thick-disk stars have been formed in regions with a relative high star-formation rate such that only Type II SNe have contributed to the chemical evolution up to $[\mathrm{Fe} / \mathrm{H}] \sim-0.5$. The "low-alpha" stars, on the other hand, come from regions with a slower star-formation rate, where 

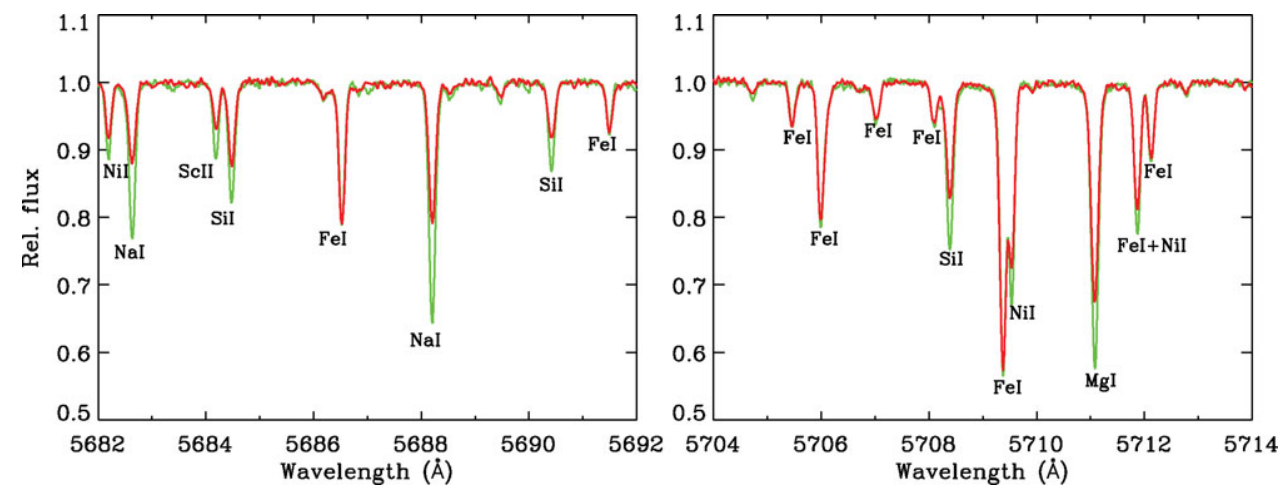

Figure 3. Spectra of two stars with nearly the same atmospheric parameters $T_{\text {eff }}, \log g$ and $[\mathrm{Fe} / \mathrm{H}]$. The spectrum of the "low-alpha" star $\mathrm{CD}-453283\left(T_{\text {eff }}=5603 \mathrm{~K}, \log g=4.57\right.$, $[\mathrm{Fe} / \mathrm{H}]=-0.89,[\alpha / \mathrm{Fe}]=0.08)$ is shown with a thick (red) line, and that of the "high-alpha" star G 159-50 $\left(T_{\text {eff }}=5648 \mathrm{~K}, \log g=4.39,[\mathrm{Fe} / \mathrm{H}]=-0.92,[\alpha / \mathrm{Fe}]=0.29\right)$ with a lighter (green) line.
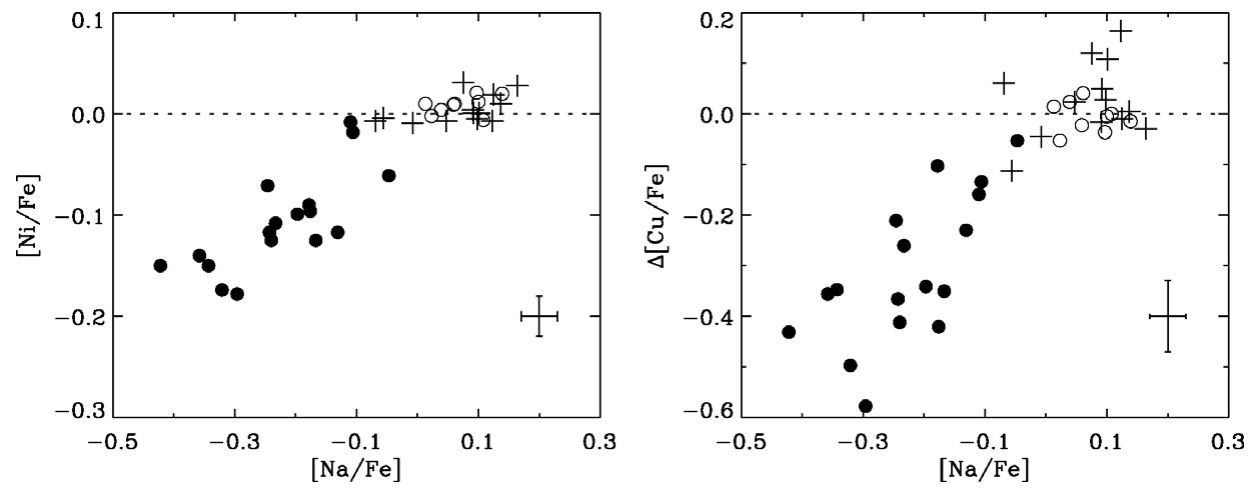

Figure 4. $[\mathrm{Ni} / \mathrm{Fe}]$ and $[\mathrm{Cu} / \mathrm{Fe}]$ vs. $[\mathrm{Na} / \mathrm{Fe}]$ with the same symbols as in Fig. 1 . In the case of $\mathrm{Cu}$ the deviation of $[\mathrm{Cu} / \mathrm{Fe}]$ from the relation defined by the thick-disk stars is plotted.

Type Ia SNe have started to contribute with iron at a metallicity $[\mathrm{Fe} / \mathrm{H}] \simeq-1.5$. The reason for the smaller separation in $[\mathrm{Ca} / \mathrm{Fe}]$ compared to $[\mathrm{Mg} / \mathrm{Fe}]$, may be that $\mathrm{Mg}$ is almost exclusively produced in Type II SNe, whereas about $25 \%$ of Ca originates in Type Ia SNe according to the chemical evolution models of Tsujimoto et al. (1995).

The abundance differences between the two halo groups can be seen directly from the observed spectra. Fig. 3 shows two spectral regions for a "high-alpha" and a "low-alpha" star with similar $T_{\text {eff }}, \log g$ and $[\mathrm{Fe} / \mathrm{H}]$ values. As seen, the Fe I lines of the two stars have nearly the same strength, whereas the $\mathrm{Mg}$ and Si lines are weaker in the "low-alpha" star. The same is the case for $\mathrm{Na}, \mathrm{Ni}$ and $\mathrm{Cu}$ lines and, as shown in Fig. 4, [Ni/Fe] and $[\mathrm{Cu} / \mathrm{Fe}]$ are well correlated with $[\mathrm{Na} / \mathrm{Fe}]$. The reason for these correlations may be that the yields of $\mathrm{Na}, \mathrm{Ni}$ and $\mathrm{Cu}$ depend on the neutron excess in supernovae and that this excess is affected by the $\alpha / \mathrm{Fe}$ ratio.

The kinematics of the stars are shown in Fig. 5. As seen from this "Toomre" diagram, both groups of halo stars have an average Galactic rotation velocity close to zero in contrast to the thick-disk stars. Furthermore, the velocity dispersion for the "low-alpha" group is higher than that of the "high-alpha" group. This larger dispersion is mainly caused by larger $U$ velocities, which means that the "low-alpha" stars tend to move on 


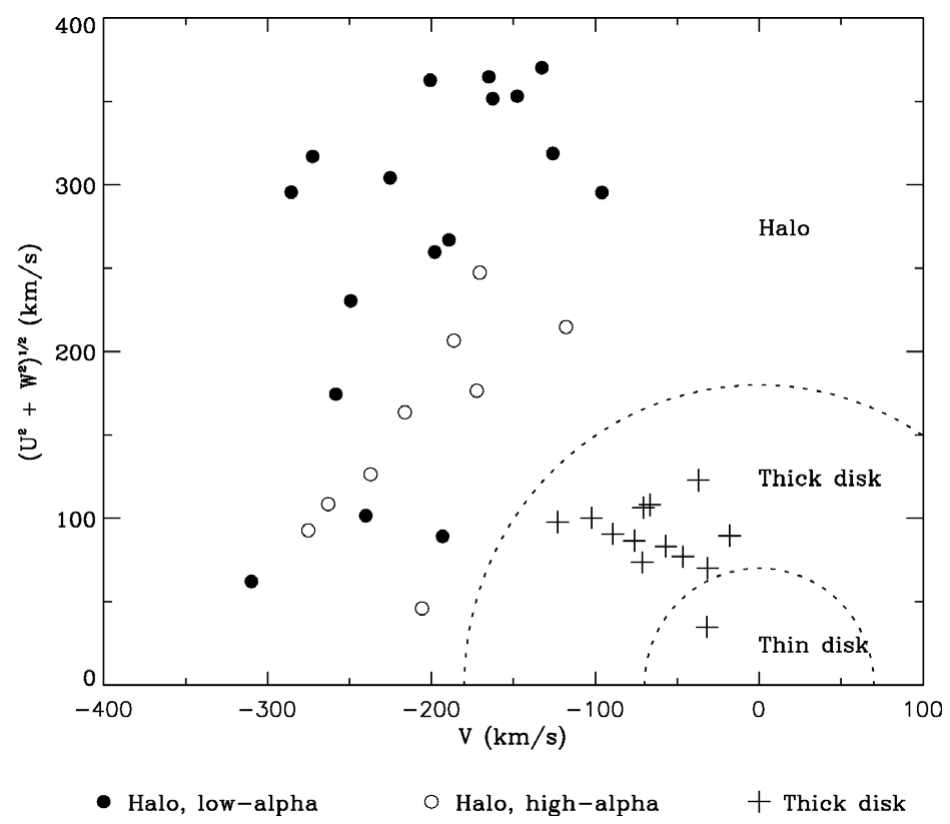

Figure 5. The "Toomre" diagram for the three groups of stars discussed in the present paper. Regions, where stars are most likely to belong to the halo, the thick disk and the thin disk, respectively (see Venn et al. 2004, Fig. 1), are separated by dashed circles corresponding to $V_{\text {total }}=180$ and $70 \mathrm{~km} \mathrm{~s}^{-1}$.

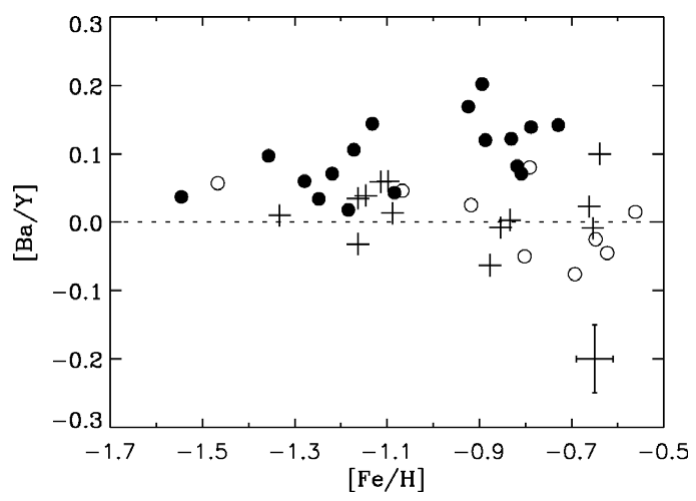

Figure 6. $[\mathrm{Ba} / \mathrm{Y}]$ vs. $[\mathrm{Fe} / \mathrm{H}]$ with the same symbols as in Fig. 1

higher energy radial orbits. A larger sample of halo stars is, however, needed before any definitive conclusions about the kinematics of the two groups can be made.

The distribution of the "low-alpha" stars in Figs. 1 and 2 shows a smooth continuation of the $\alpha / \mathrm{Fe}$ trend for thin-disk stars, which according to several studies (Reddy et al. 2003, Bensby et al. 2005) stops with $[\alpha / \mathrm{Fe}] \simeq 0.1$ at $[\mathrm{Fe} / \mathrm{H}] \simeq-0.7$. The thin-disk stars do not share, however, the low $[\mathrm{Na} / \mathrm{Fe}],[\mathrm{Ni} / \mathrm{Fe}]$ and $[\mathrm{Cu} / \mathrm{Fe}]$ values that are being found for the "low-alpha" group. In addition, the "low-alpha" stars have enhanced Ba/Y ratios as shown in Fig. 6. In all these abundance deviations, the "low-alpha" stars resemble stars in dSph galaxies (Venn et al. 2004, Sbordone et al. 2007, Koch et al. 2008) and 
in the LMC (Pompéia et al. 2007), although these satellite galaxies tend to have larger abundance offsets from disk stars than the "low-alpha" stars.

The trend of $[\mathrm{Mg} / \mathrm{Fe}]$ for the "low-alpha" halo stars in Fig. 1 agrees remarkably well with the trend predicted by Font et al. (2006, Fig. 9) from simulations of abundance distributions for a hierarchically formed stellar halo in a $\Lambda$ CDM Universe. The fact that the "low-alpha" stars do not have quite as low $[\alpha / \mathrm{Fe}]$ values as present-day satellite galaxies also agrees with their predictions. On the other hand, the "high-alpha" halo stars are not predicted from the simulations. The existence of this group suggests that the formation of the Galactic halo is more complicated than predicted from the $\Lambda$ CDM simulations. It remains to be seen if this group of "high-alpha" halo stars can be explained as due to the merger of an exceptionally large satellite or if it is a dissipative component of the Galaxy as suggested by Gratton et al. (2003). In the latter case one would expect the group of "high-alpha" stars to have some net rotation. Another problem is that the "low-alpha" halo stars tend to move on high-energy radial orbits that plunge into the Galactic central regions from the outer regions of the halo. As discussed by Gilmore \& Wyse (1998), this requires possible parent satellite galaxies to have a very high mean density to provide "low-alpha" stars on orbits with such small perigalactic distances.

In order to obtain more insight into these problems, the elemental abundances of a larger sample of metal-rich halo stars should be studied so that better knowledge of the kinematics and the relative frequency of halo stars belonging to the "high-alpha" and "low-alpha" groups can be obtained. As mentioned earlier, such data is being obtained with the Nordic Optical Telescope.

Acknowledgement. This work has been financially supported from CONACyT project 49434-F.

\section{References}

Bensby, T., Feltzing, S., Lundström, I., \& Ilyin, I. 2005, A\& $A, 433,185$

Font, A. S., Johnston, K. V., Bullock, J. S., \& Robertson, B. E. 2006, ApJ, 638, 585

Gilmore, G. \& Wyse, R. F. G. 1998, AJ, 116, 748

Gratton, R. G., Caretta, E., Desidera, S., et al. 2003, A\&A, 406, 131

Koch, A., Grebel, E. K., Gilmore, G. F. et al. 2008, AJ, 135, 1580

Nissen, P. E. \& Schuster, W. J. 1997, A\&\&A, 326, 751

Pompéia, L., Hill, V., Spite, M. et al. 2008, A $\mathscr{E} A, 480,379$

Reddy, B. E., Tomkin, J., Lambert, D. L., \& Allende Prieto, C. 2003, MNRAS, 340, 304

Reddy, B. E., Lambert, D. L., \& Allende Prieto, C. 2006, MNRAS, 367, 1329

Sbordone, L., Bonifacio, P., Buonanno, R. et al. 2007, A\& A, 465, 815

Schuster, W. J., Moitinho, A., Márquez, A., Parrao, L., \& Covarrubias, E. 2006, A $\& A$, 445, 939

Tsujimoto, T., Nomoto, K., Yoshii, Y. et al. 1995, MNRAS, 277, 945

Venn, K. A., Irwin, M., Shetrone, M. D. et al. 2004, AJ, 128, 1177 